\title{
Three-dimensional Anti-collision and Processing Simulation about Multi-axis Dual Turret Composite Wood Processing Center
}

\author{
Mingyou Chen, Heshu Xu, Kuangyu Huang, Xiangjun Zou* and Jieli Qiu \\ College of Engineering, South China Agricultural University, Guangzhou, China \\ ${ }^{*}$ Corresponding author
}

\begin{abstract}
In this paper, test on three-dimensional anti-collision and processing simulation is carried out .The test is based on modeling and virtual assembling of MSZ4224 machining center. Virtual manufacturing technology is used to finish the structure design and processing simulation of MSZ4224 machining center .This technology makes it possible to accurately analyze the quality of the product in the stage of design .By this way, disadvantages caused by traditional mechanical designing is prevented, while development cycle, costs and risks are reduced. At the same time, the capabilities of the machine are optimized. Ultimately, a method based on the idea of bounding sphere is used to discuss the anti-collision algorithm, and then research it in both time and space.
\end{abstract}

Keywords-double turret; wood processing center; processing and movement simulation; three-dimensional anti collision

\section{INTRODUCTION}

Wood processing industry products have developed to the reprocessing of finished products, and recycled products of wood from the primary processing products of logs, so that the timber industry has formed an independent production system[1].In this paper, based on the design and modeling of MSZ4224 NC machining center and the virtual assembly of the whole machine, the experiment of 3D collision prevention and machining simulation is carried out, adopts virtual manufacturing technology to complete MSZ4224 NC machining center of structure design and processing simulation analysis.

In the virtual reality environment, in order to realize the reality of virtual reality simulation system and meet the requirements of real-time collision detection, how to avoid the interference phenomenon between the simulation model is a key problem, and collision detection has become a hot topic[2].With the increase of the complexity of the object, the increase of the number of collision detection equation will lead to the increase of the computational complexity and the decrease of the detection efficiency[3].In view of the possibility of collision caused by the wrong NC codes, the simulation software of NC machine tools can be used to detect the global interference. For example, Vericut can interfere with the different parts of NC machine. However, it can only do offline simulation on the computer and is unable to provide real-time collision protection; Some foreign high-end NC machines to provide real-time collision prevention function, but it can't be applied to the existing NC machines, and the purchase of a complete set of NC machine tools and NC system product prices are very expensive[4].

Research on Virtual Machining. Lv Jinbo researched on the factory planning system and structure of virtual manufacturing[5]; Qi ruolong presented a method to realize the simulation of robot motion in virtual space[6]; Wang Wei analyzed the concept of virtual machining, and discussed the key technologies in detail[7]; Shen Chunlong studied the modeling method of NC machining center based on assembly relation and discussed its motion model[8].

The research on collision avoidance is based on virtual machining simulation, which purpose is to check the possible collision problems with the idea of simulation. The research of anti-collision based on Virtual NC machine is ultimately attributed to the interference checking of the model of virtual NC machine. Ming C. Lin proposed a kind of interference checking algorithm basing on spline model or algebraic formula, which uses algebraic method to calculate the position of interference detection; U. Benchetrit presented a method to achieve static and dynamic interference detection by the technology of hierarchical representation, and applied it to the motion simulation of the robot[9];Wang Zhong realized the interference check between the cutter and the workpiece surface by presenting the cutter with the hierarchical bounding box, while presenting the workpiece with octree[10]; Chappel put forward the a method for the accuracy test of sculptured surface, that is, representing the surface of parts by a series of discrete points, and modifying the normal vectors of discrete points by the intersecting the normal vectors of the discrete points and the swept volume of the cutter. Finally, the direction and length of normal vector represent the under-cut and over cut error of the simulation surface[11]. Takafumi Saito and Tokiichiro Takahashi proposed the $\mathrm{G}$ cache method, which takes the error of design model and simulation model in $\mathrm{Z}$ direction as the cutting error[12-13].Jerard and Drysdale have also made an in-depth study on the $\mathrm{Z}$ error verification method. They found that the errors calculated by $\mathrm{Z}$ vector are larger than those calculated by the surface normal vector. Therefore, if the machining error calculated by using $\mathrm{Z}$ vector is large, it will not be regarded as standard. On the contrary, it will only mistakes a standard one for a bad one [14-15]. 


\section{METHOD}

\section{A. Modeling of the Whole Machine}

In this paper, we have carried on modeling and simulation of the multi axis double turret composite wood processing center of the Wei De Li machinery industry Limited by Share Ltd. The render map originated from the combination of loading and unloading agencies is shown in Figure 1.

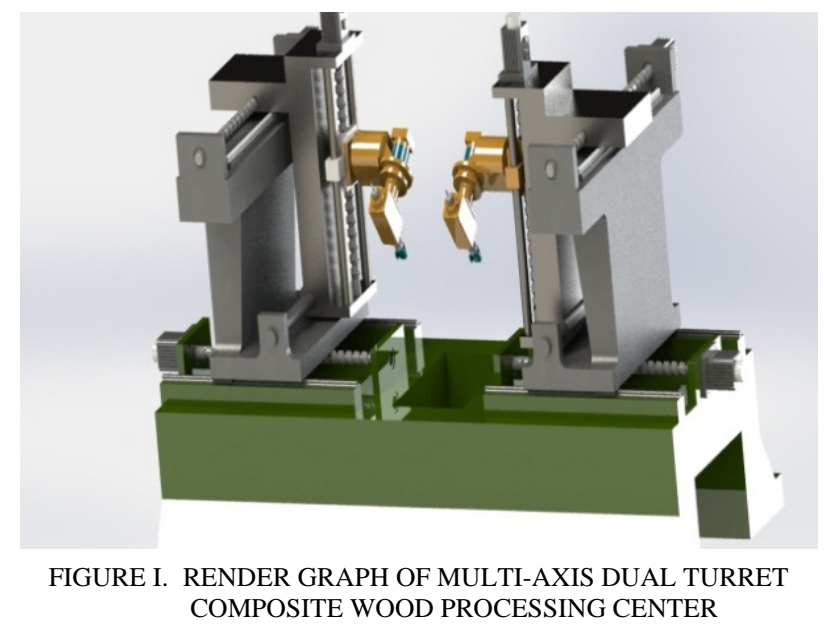

The main part of the NC machine comprises a machine body, moving axis of each direction, cutter, bench, etc. Based on the relative independence of each part of the NC machine, the composite of the machine is extracted, which is to clearly depict the various parts of the NC machine tools and the relationship among them. Components are independent to each other, and its motion characteristics are correspondent to the parts of the actual NC machine.

\section{B. Processing Simulation}

First, we selected the processing mode of mortise tenon and intermediate straight bars on both sides of the wood, and then confirm the processing route as: cutting tenon at left and right sides at the same time(Left and right cutter head are working at the same time) tenoning groove at the middle of the wood(Right cutter head processing). Finally, enable animation to achieve with Solidworks motion calculation example.

\section{Collision Detection Analysis}

Bounding sphere is the smallest sphere that contains objects, and is a bounding box of simple construction but less compact. The use of a bounding sphere will create a lot of redundant space (Except that the shape of the object is evenly distributed over all three axes).

Discrete collision detection algorithm is an algorithm that uses the similar ideas with the static interference algorithm to detect whether a collision occurs between objects at each time point $t_{1}, t_{2}, \ldots, t_{n}(n>0)$. Hubbard proposed an algorithm based on hierarchical bounding box tree, which uses the bounding sphere as a tree node to rapidly eliminate disjoint colliding objects during the intersection test. But due to the structure of the bounding sphere itself makes the tightness of the poor, in the intersection test will be a lot of unnecessary redundancy test,
So that the algorithm has some application limitations, that is, its collision detection accuracy can't be set too high.

The bounding sphere of the proposed tool is shown in Figure 2.

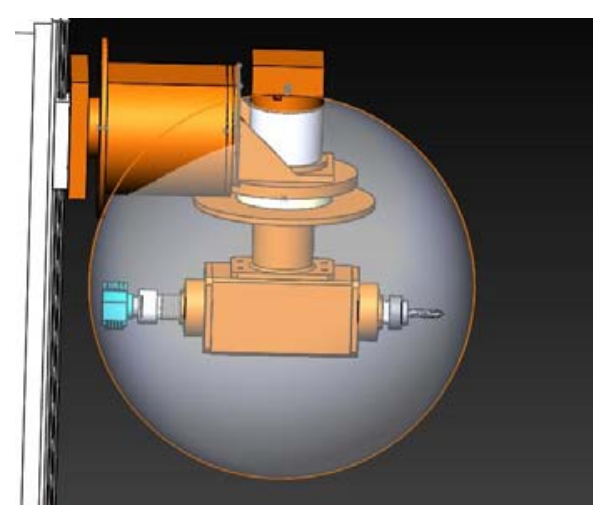

FIGURE II. THE THREE-DIMENSIONAL DIAGRAM OF THE BOUNDING SPHERE OF THE TOOL

The relative positional relationship of the component or model, and the motion relationships between the components can be determined by translating and rotating a component or model with a fixed coordinate system. After the movement of a component, the components attached to it follow the same movement, while the other components remain stationary, all of which can be expressed in a coordinate system. Both the manipulators of machine tool and the machine tool bed are associated with a coordinate system. The coordinate system 2 and coordinate system 3 of the two manipulators are located at the center of rotation of the tool rest, and the machine tool bed coordinate system 1 in the bed's right, down, front vertex. The coordinate system settings of the left and right tools are shown in Figure 3.

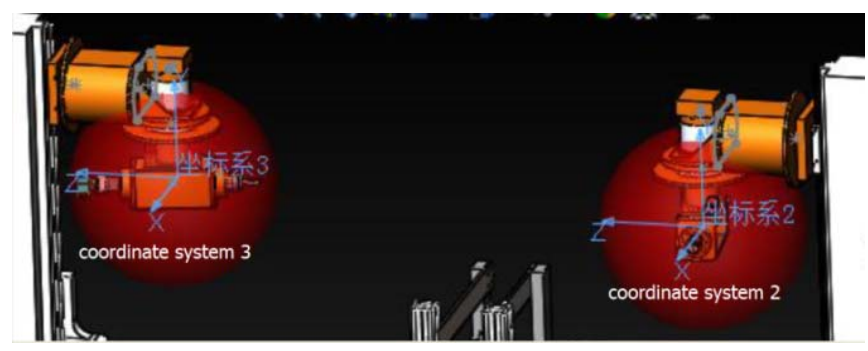

FIGURE III. THE COORDINATE SYSTEM OF TOOLS

Since the degree of freedom provided to the tool by the tool rest is the degree of freedom of rotation, and according to the coordinates setting of Figure 3, we can see whether the left and right two spheres have interference mainly depends whether there are cross points on the linear coordinate map of the $\mathrm{z}$ direction. So as long as compare and analyze the range bands in the $\mathrm{z}$ direction of both sides, we can explain whether there are collisions in the processing center in the processing simulation. The comparison results are shown in Figure 4. 


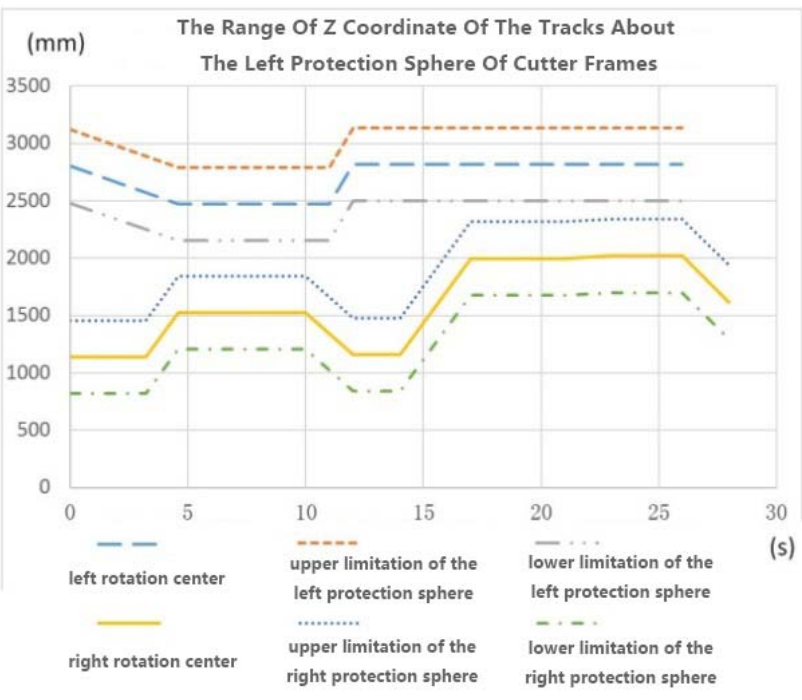

FIGURE IV. THE RANGE OF Z COORDINATE OF THE TRACKS ABOUT THE LEFT PROTECTION SPHERE OF CUTTER FRAMES

Through the simulation, we can clearly see from the figure, the range bands in the $\mathrm{z}$ direction of the left and right tool rest protection sphere do not coincide. It means that the two tool rests do not collide with each other during the machining process.

\section{Three-Dimensional Anti-Collision Analysis}

The calculation of the bounding sphere is relatively simple compared to other bounding boxes: the average of the three coordinate values ( $\mathrm{x}, \mathrm{y}$ and $\mathrm{z}$ ) of all the basic geometric elements is the center of the bounding sphere $\mathrm{c}(\mathrm{x}, \mathrm{y}, \mathrm{z})$. The maximum distance between the centers of the sphere to each basic element in an object is the radius $\mathrm{R}$ of the bounding sphere.

It is easy to know by the configuration of the bounding sphere described above that the bounding sphere's storage structure relative to other bounding box's storage structure is relatively simple and it only need four 4-byte floating point number (Description of the center of the sphere needs 3 floating-point numbers, and the description of radius needs 1 floating-point number).

This intersection test method only needs to calculate whether the distance between the two spheres is less than the sum of their radius. If it is less than the sum of their radius, it means that a collision may occur. Compared with other bounding box intersection test method, the intersecting test method of bounding sphere is relatively simple. The comparison formula is as follows:

$$
\sqrt{\left(x_{1}-x_{2}\right)^{2}+\left(y_{1}-y_{2}\right)^{2}+\left(z_{1}-z_{2}\right)^{2}}<R_{1}+R_{2}
$$

$\mathrm{x}_{1}, \mathrm{y}_{1}$ and $\mathrm{z}_{1}$ are the three coordinates of the center of rotation of the left side; $\mathrm{x}_{2}, \mathrm{y}_{2}$ and $\mathrm{z}_{2}$ are the three coordinates of the center of rotation of the right side; $R_{1}$ and $R_{2}$ are the radius of the left and right bounding spheres, respectively.
Since the bounding sphere always surrounds the object, it is contained within the bounding sphere, regardless of how the object rotates. Therefore, bounding sphere only needs to carry out the corresponding coordinate translation to the center of sphere in the course of the movement of objects.

According to equation (1) to determine when the two turrets' bounding spheres without intersection, indicating that no collision may occur. When there is intersection, it indicating the possibility of a collision. In this case, the bounding spheres need to be further broken down and then repeated until the set accuracy is reached. If the beginning does not interfere, indicating that the two objects of the study did not collision. If there is interference, as there is a large amount of redundancy in the first-order bounding sphere, it does not necessarily contain the components of the object to be studied. So it is necessary to analyze whether or not the interfering bounding sphere contains the parts of the two objects to be studied. If not, continue the loop. If so, exit, and get the results of the collision process of movement. Figure 5 is a flow chart of a collision detection algorithm.

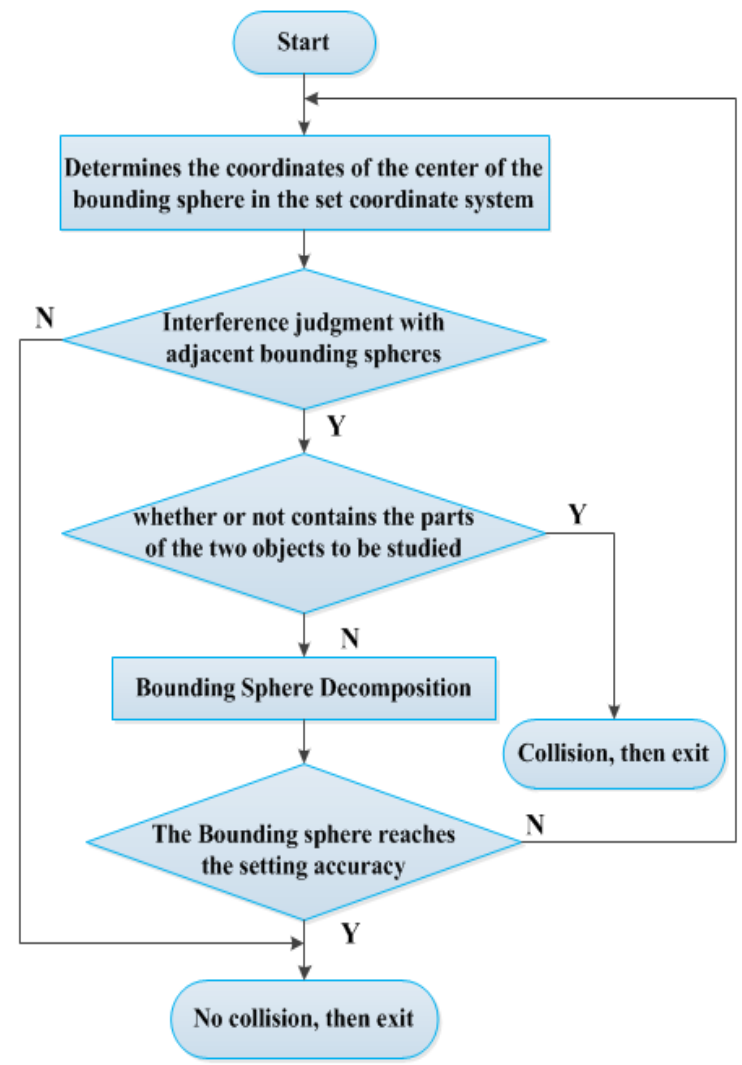

FIGURE V. COLLISION DETECTION ALGORITHM FLOW CHART

\section{SimULATION RESULTS}

Figure 6 shows the tool rest of the two sides simultaneously moved closer from both sides and the distance of the tool rests is shrinking. 


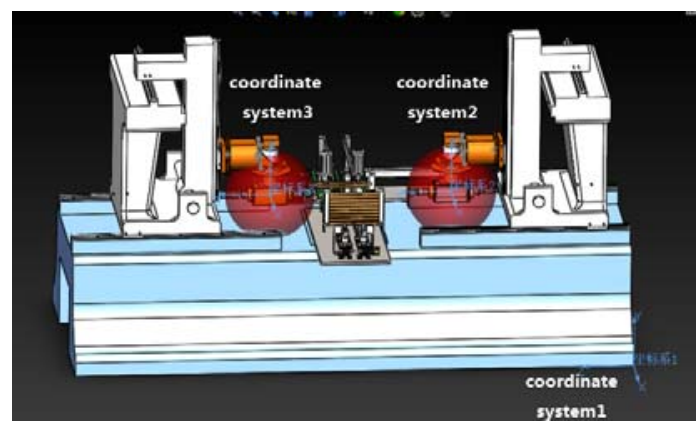

FIGURE VI. THE LEFT AND RIGHT TOOL REST MOVE CLOSER GRADUALLY

Figure 7 shows the tool rests of two sides are processing both sides of the tenon of wood. At this time the distance between the two centers is the smallest distance, the value is $949.61 \mathrm{~mm}$. The radius of the two central spheres is $320 \mathrm{~mm}$ and according to equation (1), because of 949.61> 640, two spheres did not intersect. With the algorithm of bounding sphere, the conclusion is no collision.

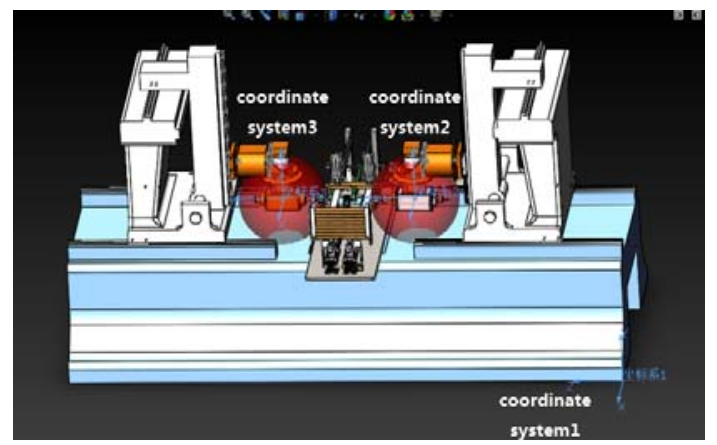

FIGURE VII. THE TOOL RESTS OF TWO SIDES ARE PROCESSING BOTH SIDES OF THE TENON OF WOOD

\section{CONCLUSION}

The research object of this paper is MSZ4224 NC machining center. Based on the design modeling and virtual assembly of the whole machine in the MSZ4224 NC machining center, the three dimensional anti-collision and machining simulation experiments were carried out. Design and machining simulation analysis to MSZ4224 NC machining center were carried out using virtual manufacturing technology. The design quality of the product can be accurately analyzed at the product design stage, which to avoid the unfavorable factors caused by use of traditional mechanical design methods to develop machine tools. It shortens the product development cycle, reducing development costs and product development risks, and also makes the performance of the machine to achieve optimal.

What anti-collision used is the bounding sphere method in virtual reality. Firstly, the moving coordinate map of the bounding sphere is obtained with the discrete collision detection algorithm, and the motion interference result is obtained by the graphical comparison. Then, the feasibility of anti-collision algorithm is discussed by hierarchical bounding box method. The anti-collision detection algorithm is discussed both in time and space. The simulation results show that the simulation method described in this paper can effectively simulate the processing situation, with production value and practical significance.

\section{ACKNOWLEDGEMENTS}

This work was financially supported by the National Natural Science Foundation of China (No.31571568), Supported by Science and Technology Planning Project of Guangdong Province.(No.2014A010104011), science and technology project of Guangzhou city (No.201510010140), science and technology project of Huizhou city (No.2014B040008006). Corresponding Author: Xiangjun Zou.

\section{REFERENCES}

[1] Wang ZB, Zhu XW, He S, et al. Review of the Development of NC Woodworking Machinery. Forestry Machinery and Woodworking Equipment, 2012, 40(11):8-13.

[2] Jang GY. Research and application of collision detection algorithm based on bounding volume. Chengdu: University of Electronic Science and Technology of China, 2012.

[3] Canny, J. Collision Detection for Moving Polyhedral. IEEE Transaction on Pattern Analysis, 1986, 18(2): 200-209.

[4] Lu Y. Motion simulation and interference check of multi-axis NC machine tools. Harbin: Harbin Institute of Technology, 2015.

[5] Lv JB, Meng XF. Application status of virtual manufacturing technology in the factory planning. Machinery Maintenance Management, 2014, 27(6): 118-120.

[6] Qi Rl, Zhou WJ, Liu JG, et al. An effective method for implementing virtual Control and 3D simulation of robot motion in VC platform. Robot, 2013, 35(5): 594-599.

[7] Wang W, Zhang P, Liu QY. Research in virtual simulation technology development in manufacturing industry. Modular Machine Tool and Automatic Manufacturing Technique, 2001, 13(10): 1-4.

[8] Shen CL, Zhang YL. Research on 3D virtual machining environment and key technology. Journal of Computer-Aided Design and Computer Graphics. 2001, 13(10): 890-894.

[9] Benchetrit U, Lenz E, Shoham M. Collision detection in static and dynamic complex scenes not having geometry coherence. The International Journal of Advanced Manufacturing Technology, 1998, 14(1): 70-76.

[10] Wang Z. Research on 5-axis NC machining interference inspection technology. Hangzhou: Zhejiang University, 2005.

[11] Chappel L.T. The use of vector to simulate material removed by numerically controlled milling. Computer-Aided Design, 1983, 15(3): 156-158.

[12] Takafumi S, Tokiichiro T. Comprehensible rendering of 3D shapes. Computer Graphics, 1990, 24(4): 197-206.

[13] Takafumi S, Tokiichiro T. NC machining with G-Buffer method. Computer Graphics, 1991, 25(4): 207-216.

[14] Jerard R B, Drysdale R L, Hauck K, et al. Methods for detecting errors in numerically controlled machining of sculptured surfaces. IEEE Computer Graphics and Applications, 1989(1): 26-39.

[15] Drysdale, RB Jerard, B. Schaudt, et al. Discrete Simulation of NC Machining. Algorithmic Special Issue on Computational Geometry, 1989, 4 (1): 33-60. 